\title{
A MEMS Thermal Biosensor for Metabolic Monitoring Applications
}

\author{
Li Wang, David M. Sipe, Yong Xu, Member, IEEE, and Qiao Lin
}

\begin{abstract}
This paper presents a microelectromechanical systems (MEMS) differential thermal biosensor integrated with microfluidics for metabolite measurements in either flow-injection or flow-through mode. The MEMS device consists of two identical freestanding polymer diaphragms, resistive heaters, and a thermopile between the diaphragms. Integrated with polymer-based microfluidic measurement chambers, the device allows sensitive measurement of small volumes of liquid samples. Enzymes specific to a metabolic analyte system are immobilized on microbeads packed in the chambers. When a sample solution containing the analyte is introduced to the device, the heat released from the enzymatic reactions of the analyte is detected by the thermopile. The device has been tested with glucose solutions at physiologically relevant concentrations. In flow-injection mode, the device demonstrates a sensitivity of approximately $2.1 \mu \mathrm{V} / \mathrm{mM}$ and a resolution of about $0.025 \mathrm{mM}$. In flow-through mode with a perfusion flow rate of $0.5 \mathrm{~mL} / \mathrm{h}$, the sensitivity and resolution of the device are determined to be approximately $0.24 \mu \mathrm{V} / \mathrm{mM}$ and $0.4 \mathrm{mM}$, respectively. These results illustrate that the device, when integrated with subcutaneous sampling methods, can potentially allow for continuous monitoring of glucose and other metabolites.

[2007-0085]

Index Terms-Biological thermal factors, biosensors, enzymatic reactions, glucose detection, metabolic monitoring, microelectromechanical devices, microelectromechanical systems (MEMS) thermal sensors, thermal transduction, thermoelectric devices, transducers.
\end{abstract}

\section{INTRODUCTION}

$\mathbf{T}$ HERMAL biosensors measure thermal energy released or absorbed in biochemical reactions. Thermal activities exist ubiquitously in biological processes, and thermal biosensing is hence a widely applicable method. Requiring no labeling of reactants, thermal biosensing is a universally useful method [1], allowing direct interrogations of elementary processes in biochemistry without sophisticated cascades of reaction steps required by other signal transduction methods. However, conventional thermal biosensors are in general rather complicated in construction and require large sample volumes (e.g., milliliters).

Manuscript received April 18, 2007; revised October 22, 2007. This work was supported in part by the National Science Foundation under Award DBI0650020. Subject Editor S. Shoji.

L. Wang is with the Department of Mechanical Engineering, Carnegie Mellon University, Pittsburgh, PA 15213-3890 USA.

D. M. Sipe is with the Department of Chemistry, Carnegie Mellon University, Pittsburgh, PA 15213-3890 USA.

$\mathrm{Y} . \mathrm{Xu}$ is with the Department of Electrical and Computer Engineering, Wayne State University, Detroit, MI 48202 USA.

Q. Lin is with the Department of Mechanical Engineering, Columbia University, New York, NY 10025 USA (e-mail: qlin@ columbia.edu).

Color versions of one or more of the figures in this paper are available online at http://ieeexplore.iee.org.

Digital Object Identifier 10.1109/JMEMS.2008.916357
Microelectromechanical systems (MEMS) technology allows batch fabrication and integration of miniaturized devices at low cost, and has been used to create a variety of integrated bioanalytical devices. In particular, MEMS thermal biosensors, because of their improved thermal isolation as well as reduced thermal mass and sample volume, offer improved sensitivity and linear range, reduced power consumption and shortened measurement times. When batch-fabricated in arrays, such devices may also enable high-throughput operation in which multiple samples are measured in parallel.

MEMS thermal sensors are often based on temperature detection. Temperature sensors can typically be either thermistors or thermopiles. Thermistors, which rely on changes in their electric resistance with temperature, allow measurement of absolute temperatures but in general are limited in sensitivity [2]. A thermopile, which is a set of thermocouple junctions connected in series, allows measurement of differences in temperature between two regions where the thermocouple junctions are located. In general, thermopiles allow excellent common-mode noise cancellation and zero offset, and therefore can be highly sensitive [3]. Thin film thermopiles have been fabricated from semiconductors [4]-[6], metals [7], [8] and semiconductor compounds [9] on various substrates. To maximize thermal isolation and reduce thermal mass, suspended diaphgrams or bridges have been used, which have achieved nanojoule or nanowatt thermal detection sensitivities [10] and time responses approaching microseconds [11]. Such thermal components have been used in infrared detection [4], [11], material characterization [12] and gas sensing [13], [14].

The investigations above have demonstrated the promise of MEMS thermal sensors. However, they are typically limited to solid or gas samples, and do not allow measurement of liquid samples as generally required by biosensors. Research into MEMS thermal sensors for liquid samples has been relatively limited, and has involved detection of glucose [2], [5], [6], [15], uric acid [16], and insulin [17], as well as studies of living systems such as microorganisms, human or animal cells and tissues [8], [18], [19]. Multianalyte thermal biosensors using MEMS thermopiles have also been investigated [2], [20], [21]. These devices share a major limitation in the use of conventionally constructed measurement flow cells [4], [5]. As such, they lack proper microfluidic handling capabilities, and require large flow cell volumes ranging from $10 \mu \mathrm{l}$ to around $1 \mathrm{~mL}$ [2], [5], [7], [8], [18]. In a recent attempt to allow smaller liquid sample volumes, Zhang and Tadigadapa [6] developed a glucose sensor which used a microfluidic chamber fabricated from glass or polydimethylsiloxane (PDMS). However, the device required high-temperature processing because of its use of solid-state 
diaphragms and temperature sensors. Also, its relatively low sensitivity ( $\sim 1 \mathrm{mM}$ of detectable glucose concentration), which may have arisen from the significant noise in its semiconductor thermopile, is inadequate for practical applications [22]-[24].

This paper describes a MEMS differential thermal biosensor that explores the integration of microfluidics with thermal sensing to allow sensitive measurement of small volumes of analyte solutions. Specifically, a polymer microfluidic structure is integrated with a silicon-based thermal sensor chip. The microfluidic structure consists of chambers and channels for handling and measuring drastically reduced biological sample volumes $(0.8 \mu \mathrm{l})$. The integration of a bimetallic thermopile on the microfluidic structure allows low-noise measurement of sample temperature, and the use of the differential measurement approach enables excellent common-mode noise cancellation, leading to significantly improved sensitivity. We will demonstrate our device with enzymatic glucose detection. Due to its importance in the management of diabetes and other diseases, glucose sensing has long been a focus of biosensor research and development, including MEMS-based systems using electrochemical [25], [26], electrophoretic [27], optical [28], colorimetric [29], volumetric [30], and viscometric [31] detection methods. The present device will complement these systems, and yield significant improvements over existing thermally based MEMS glucose sensors as mentioned above. When combined with MEMS microdialysis components [32], it will potentially result in an integrated, minimally invasive glucose monitoring system.

This paper is organized as follows. We first describe the device design (Section II) and operating principle (Section III). We then present the device fabrication process (Section IV) and testing method (Section V). Calibration and metabolite measurement results are presented in Section VI, which is followed by concluding remarks in Section VII.

\section{DESIGN}

A schematic of the MEMS thermal biosensor is shown in Fig. 1. The device primarily consists of a thermal sensor chip integrated with a microfluidic system featuring two identical chambers. The chambers are filled with microbeads whose surfaces are functionalized with enzymes specific to the analyte to be measured. During device operation, an analyte sample solution and a reference buffer solution are respectively loaded into the chambers. Enzyme-catalyzed reactions of the analyte produce a thermal power, which can be measured via the temperature difference between the sample and reference chambers. This differential measurement affords excellent cancellation of common mode disturbances in the two chambers, such as room temperature fluctuations, viscous energy dissipation, and thermal activities of nontarget molecules present in the solutions. Thus, highly sensitive measurement of the reaction heat can be accomplished. The device can be used either in flow-injection mode, in which the analyte and reference solutions are injected in a prescribed volume and then the reaction occurs in the absence of fluid flow, or flow-through mode, in which the solutions are continuously perfused through the chambers.

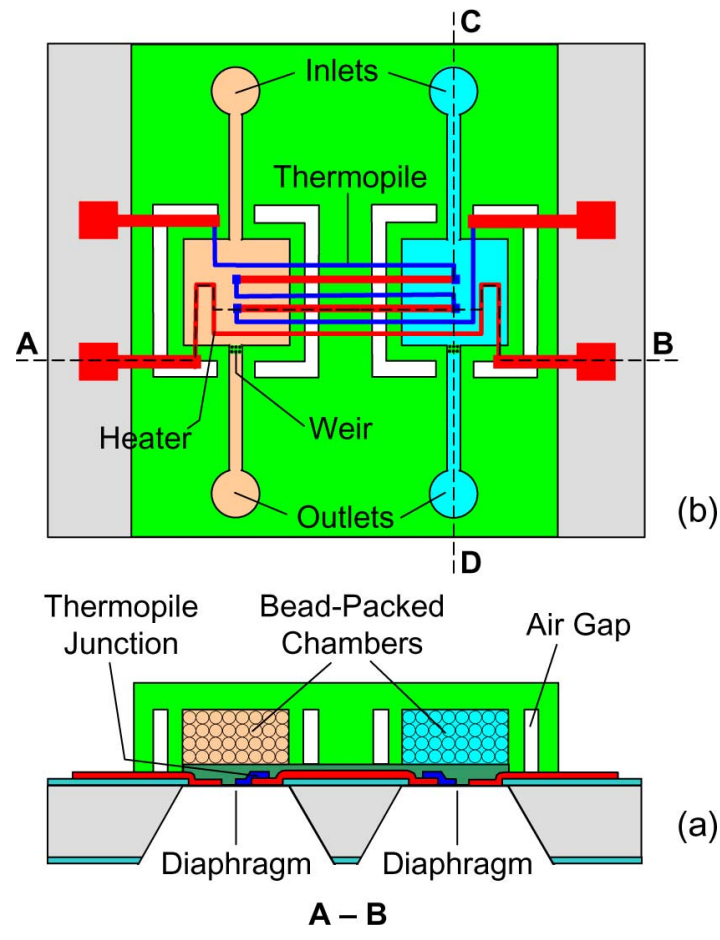

Fig. 1. Schematic of the MEMS thermal biosensor: (a) Top view and (b) cross-sectional view as defined by the line A-B indicated in the top view.

In the microfluidic system, the chambers are each connected to inlets and outlets by microchannels, which allow effective handling of small volumes of analyte and reference solutions. The enzyme-functionalized microbeads are also loaded into the chambers using the inlet channels, and are trapped in the chambers by a weir structure located at the junction of each chamber and its outlet channel. An important feature of the microfluidic system is that the chambers are each based on a freestanding polymer diaphragm. The freestanding design as well as the low thermal conductivity of polymers significantly improve the thermal isolation of the chambers. Thus, for a given reaction power, a significant temperature difference can be generated between the chambers, leading to improved device sensitivity. The freestanding diaphragm also reduces the thermal mass of the chambers. The chambers' thermal isolation is further increased, and their thermal mass further reduced, by surrounding the chambers with air gaps incorporated in the microfluidic system. Because of the minimized thermal mass, the device can have a time response sufficiently fast to allow real-time measurements in either flow-injection or flow-through mode.

The temperature difference between the sample and reference chambers is detected by a thermopile integrated on the diaphragms. The thermopile consists of thin-film chromium and nickel junctions, with the hot and cold junctions respectively located on the diaphragms. The temperature difference induces a voltage in the thermopile, which is the device's direct output. Compared with semiconductor thermopiles, the bimetallic thermopile allows low temperature processing appropriate for polymers and allows for much reduced noise levels. Metal resistive microheaters are included primarily for device calibration. Additionally, these heaters may be used to maintain the sample and reference solutions at desired temperatures. 
We envision that the MEMS thermal biosensor will be useful for minimally invasive monitoring of glucose and other metabolites in medical applications. For example, the device could be coupled to a subcutaneous sampling method, such as microdialysis. In microdialysis, a sampling probe is inserted into subcutaneous tissue. A small channel is embedded in the probe and separated from the tissue by a semipermeable membrane. A buffer is perfused through the channel, samples glucose in the interstitial fluid through the membrane, and then emerges at the channel exit. The outflowing buffer could be fed directly into the MEMS thermal biosensor, which can directly detect the sampled glucose by operating in flow-through mode. Alternatively, the outflowing buffer could be collected in a fraction collector, and injected as a batch into the MEMS sensor, which then detects the glucose in flow-injection mode. This procedure can potentially be accomplished by a microsystem that integrates the MEMS sensor with a microfabricated microdialysis probe [32], [33] and a fraction collector [34].

\section{OPERATING PRINCIPLE}

Let an analyte sample (e.g., glucose or other metabolites), at concentration $c_{\mathrm{s} 0}$, and a reference buffer, be introduced into the two device chambers, respectively. Catalyzed by an enzyme, the analyte is reacted, so its concentration $c_{\mathrm{s}}$ decreases in time from $c_{\mathrm{s} 0}$. Typically, the device operates in a range of low $c_{\mathrm{s} 0}$ so that enzyme activity is unsaturated [35]. In the limiting case where $c_{\mathrm{s} 0}$ is small compared with $K_{\mathrm{M}}$, the enzyme's Michaelis constant, the rate at which the analyte reacts is given by a linear relationship [35]

$$
v=\lambda c_{\mathrm{s}} \quad \text { with } \quad \lambda=k_{\mathrm{cat}} c_{\mathrm{e} 0} / K_{\mathrm{M}}
$$

where $k_{\text {cat }}$ is the enzyme's turnover number, and $c_{\mathrm{e} 0}$ is the total effective enzyme concentration in the chamber. Note that $1 / \lambda$, with units of time, represents the time scale of the enzymatic reaction. The reaction power per unit volume is thus given by

$$
\dot{q}=v \Delta H
$$

where $\Delta H$ is the enthalpy change of the enzymatic reaction per mole of analyte reacted.

When the device operates in flow-injection mode, the analyte concentration can be assumed to be uniform in the entire chamber and decrease in time according to the relationship $d c_{\mathrm{s}} / d t=-\lambda c_{\mathrm{s}}$, where (1) has been used. This differential equation can be solved to obtain

$$
c_{\mathrm{s}}=c_{\mathrm{s} 0} e^{-\lambda t}
$$

When the device operates in flow-through mode, the sample and reference solutions are each perfused through the chambers at a certain flow rate, $Q$. For simplicity, assume that the flow in the chamber is 1-D with cross-sectional area $A$ and length $L$. A consideration of mass balance yields the differential equation $d c_{\mathrm{s}} / d x=-(\lambda A / Q) c_{\mathrm{s}}$. This equation can be solved to obtain the analyte concentration, which is a function of time given by

$$
c_{\mathrm{S}}=c_{\mathrm{S} 0} e^{-(\lambda A / Q) x} .
$$

Thus, the reaction power density in flow-injection and flowthrough modes can be obtained by substituting (3) or (4) into (1), which is in turn substituted into (2). The total reaction power in the sample chamber $P_{\mathrm{s}}$ is obtained by integrating the power density over the entire chamber volume. Because the power in the reference chamber, in which there is no reaction, is $P_{\mathrm{r}}=0$, the total differential power, $\Delta P=P_{\mathrm{s}}-P_{\mathrm{r}}=\int_{V} \dot{q} d V$, between the two chambers can thus be obtained

$$
\Delta P= \begin{cases}c_{\mathrm{s} 0} \lambda V \Delta H e^{-\lambda t} & (\text { flow - injection mode) } \\ c_{\mathrm{s} 0} Q \Delta H\left(1-e^{-\lambda V / Q}\right) & (\text { flow - through mode })\end{cases}
$$

In the special case of small flow rates such that the residence time of the solution in the chamber is much larger than the reaction time constant (i.e., $V / Q \gg 1 / \lambda$ ), linearizing the flow-through mode equation yields the following limiting-case relationship:

$$
\Delta P=c_{\mathrm{s} 0} Q \Delta H \text { (flow-through mode, small flow rate). }
$$

That is, the total differential power is proportional to the analyte concentration at small flow rates.

The differential power causes the temperatures of the sample $\left(T_{\mathrm{S}}\right)$ and reference $\left(T_{\mathrm{r}}\right)$ chambers to differ. For simplicity, we consider a lumped-parameter, steady-state thermal model for the device, which relates the temperature difference, $\Delta T=$ $T_{\mathrm{s}}-T_{\mathrm{r}}$, to the differential power by a linear relationship [36]

$$
\Delta T=R_{\mathrm{eff}} \Delta P \quad \text { with } \quad R_{\mathrm{eff}}=R_{\mathrm{th}} /\left(1+2 \rho c_{\mathrm{p}} Q R_{\mathrm{th}}\right)
$$

where $R_{\text {eff }}$ is the effective thermal resistance, $R_{\mathrm{th}}$ is the thermal resistance between the chambers in the absence of flow (i.e., in flow-injection mode), and $\rho$ and $c_{\mathrm{p}}$ are the density and specific heat of the solutions. Note that the steady state assumption would be appropriate when the device's thermal time constant, $\tau=R_{\mathrm{th}} C_{\mathrm{p}}$, where $C_{\mathrm{p}}$ the effective thermal capacity of each chamber, is small compared with the reaction time constant $1 / \lambda$.

The temperature difference induces a voltage in the thermopile between the chambers: $\Delta U=n S \Delta T$, where $n$ is the number of thermocouples in the thermopile, $S$ is the Seebeck coefficient, i.e., the voltage in one pair of thermocouple junctions in response to a unit temperature difference. From (7), the thermopile voltage is thus given by

$$
\Delta U=K \Delta P \quad \text { with } \quad K=n S R_{\text {eff }}
$$

where $K$ is the device's responsivity. Therefore, one can use (8) to determine the differential power from the measured thermopile voltage, which then allows determination of the analyte concentration from (5).

\section{FABRication Process}

The fabrication process (Fig. 2) started with a silicon wafer with both sides covered with thermally grown silicon dioxide $(1 \mu \mathrm{m})$. The frontside oxide was patterned to define the insulation area for electrical contacts using buffered hydrogen fluoride. Chromium $(0.5 \mu \mathrm{m})$ and nickel $(0.2 \mu \mathrm{m})$ were then 


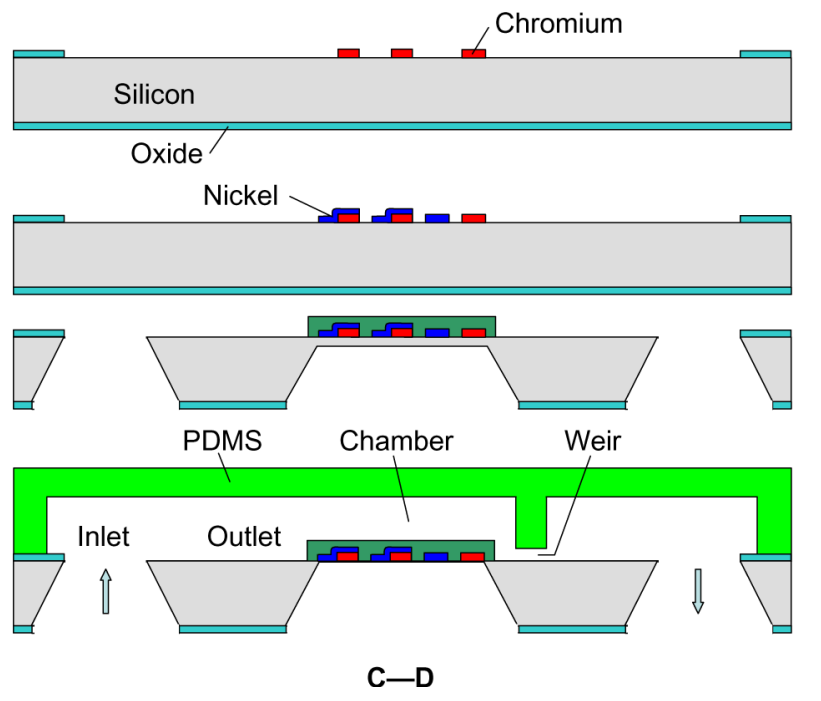

Fig. 2. Fabrication process illustrated using the cross section C-D as indicated in Fig. 1(a). (a) Chromium and (b) nickel deposition and patterning. (c) SU-8 coating and patterning. (d) Diaphragm release and bonding of the thermal sensor chip with a PDMS sheet.

deposited via sputtering and patterned to form the integrated resistive heaters (nickel), thermopile (nickel/chromium) and bonding pads (chromium) [Fig. 2(a) and (b)]. Next, tetramethyl ammonium hydroxide was used to etch the wafer from its backside with the oxide there patterned as an etching mask, until there was approximately $50 \mu \mathrm{m}$ of silicon left in the diaphragm area on the frontside. An SU-8 thin film $(5-\mu \mathrm{m})$ was spin-coated and patterned to form the diaphragms $\left(2 \times 2 \mathrm{~mm}^{2}\right)$ [Fig. 2(c)], which upon wafer dicing were made freestanding by gas-phase $\mathrm{XeF}_{2}$ etching of the silicon layer underneath [Fig. 2(d)].

The microfluidic chip was fabricated from PDMS by replica molding [37]. To fabricate the mold, $6.5 \mu \mathrm{m}$ of SU-8 was spincoated on a silicon wafer and exposed to UV to define the weir structure. Without developing this layer, a second SU-8 layer (200- $\mu \mathrm{m}$ thick) was spin-coated and UV-exposed to define the chambers and channels. The SU-8 mold was completed by developing away the unexposed SU-8. Then, PDMS was cast onto the SU-8 mold, cured at $70{ }^{\circ} \mathrm{C}$ for $3 \mathrm{~h}$ and peeled off to obtain a sheet bearing the microfluidic features including the chambers and channels (200 $\mu \mathrm{m}$ in height), as well as the bead-trapping weirs $(6.5 \mu \mathrm{m}$ in height). The PDMS sheet was next bonded to the silicon chip's frontside after oxygen plasma treatment of the bonding surfaces [Fig. 2(d)]. The fluidic interface to the macroworld was made by inserting Tygon tubing (inside diameter: $0.5 \mathrm{~mm}$; outside diameter: $1.5 \mathrm{~mm}$ ) into the inlet and outlet wells on the backside and sealed with epoxy. The photographs of a thermal biosensor chip before and after PDMS bonding and packaging are shown in Fig. 3.

\section{Materials And Test Setup}

Phosphate buffered saline without calcium or magnesium (CMF-PBS) was used as the reference solution in metabolite measurements, as well as to fill the sample chamber in device calibration. Glucose oxidase (Aspergillus niger,
$296 \mathrm{U} \cdot \mathrm{mg}^{-1}$, Sigma-Aldrich) and catalase (bovine liver, $47000 \mathrm{U} \cdot \mathrm{mg}^{-1}$, Sigma-Aldrich,) were immobilized, using streptavidin-biotin chemistry, on $9.77 \mu \mathrm{m}$ polystyrene microbeads (SuperAvidin ${ }^{\mathrm{TM}}$ coated microspheres, Bangs Laboratories) as follows. The beads were washed twice with and then resuspended in CMF-PBS containing 0.1\% BSA and 0.05\% Tween-20. Solutions of biotin-conjugated GOX and CAT at $1.54 \mathrm{mg} / \mathrm{mL}$ and $0.96 \mathrm{mg} / \mathrm{mL}$ concentrations, respectively, were prepared. Next, the enzymes were mixed in a 1:1 mass ratio, added to the beads, such that the combined total number of the enzymes exceeds the total number of binding sites on the beads by a ratio of 2000:1. The resulting mixture was incubated overnight at room temperature. The beads were finally rinsed thoroughly with and resuspended in CMF-PBS containing $0.1 \%$ BSA and $0.05 \%$ thimerosal for use in testing. Also for testing use, $\mathrm{D}(+)$-glucose (Sigma-Aldrich) was dissolved in CMFPBS at selected concentrations. The solutions were degassed by a vacuum chamber prior to all measurements.

The device was tested using a setup as shown in Fig. 4. A program in LabVIEW (National Instruments) was developed for experiment automation and data acquisition during calibration and measurement. A syringe pump (KDS200, KD Scientific) was used to load microbeads as well as sample and buffer solutions into the chambers. The thermopile output was measured by a nanovoltmeter (34420A, Agilent), which was automated by a PC using LabVIEW. On-chip heaters were connected to a dc power supply (E3631A, Agilent).

Enzyme-functionalized microbeads were packed into the chambers through microfluidic channels. The densely packed beads and the narrow gaps in the weir required hydrodynamic driving pressures up to a few tens of kilopascal. A pressure gauge was used for pressure monitoring, ensuring that excessive pressures were avoided on the diaphragms. During calibration, a known thermal power was imparted to the sample chamber by Joule heating of the microheaters, while the resulting thermopile output was recorded. During metabolite measurements, the sample and reference solutions were either injected or flowed through the chambers to interact with the enzyme on the bead surfaces, where the resulting thermopile output was obtained.

\section{RESUlts AND Discussion}

\section{A. Device Calibration}

We first characterized properties of the thin-film nickel heaters and the chromium-nickel thermopile on the freestanding polymer diaphragms. Infrared thermometry (Infrascope II, QFI) was used to measure temperature distributions (Fig. 5) on a diaphragm heated by thin-film heaters. Temperature variations on the diaphragm were found to be smaller than $1{ }^{\circ} \mathrm{C}$. Temperature measurements by IR thermometry were also used to calibrate the thermoelectric response of the thermopile (Fig. 6). It can be seen, as expected from thermoelectric theory [38], that the thermopile output is highly linear with the temperature difference between the thermopile junctions located respectively on the two diaphragms. The Seebeck coefficient is determined to be $37 \mu \mathrm{V} / \mathrm{K}$ from this linear 


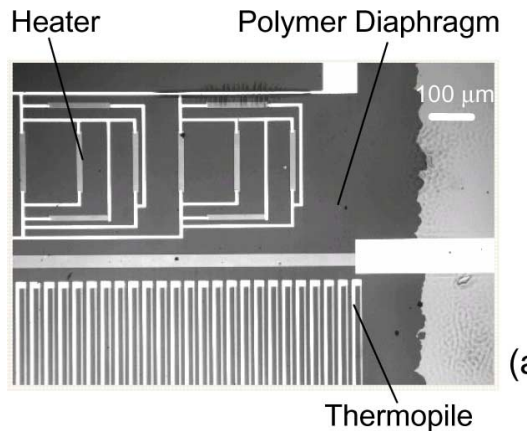

(b)

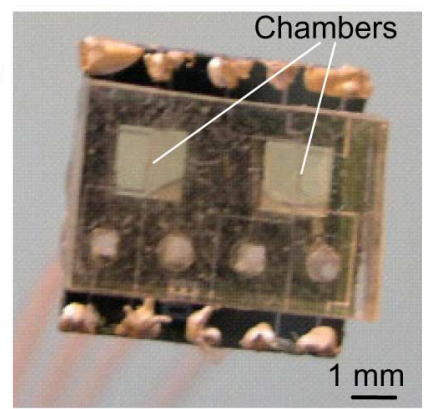

Fig. 3. Fabricated MEMS thermal biosensor. (a) Micrograph of the thermal sensor chip. (b) Photograph of the fully packaged device.

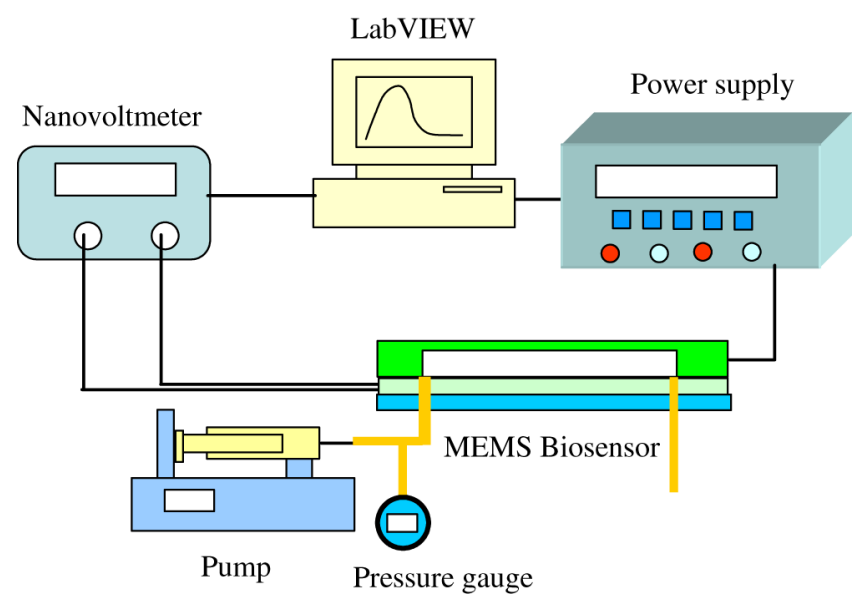

Fig. 4. Experimental setup with automated measurements controlled via LabVIEW.

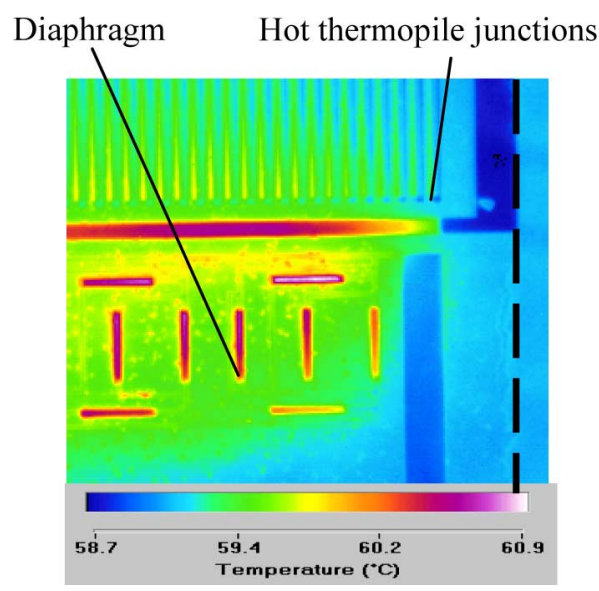

Fig. 5. Temperature map on a portion of the diaphragm by IR thermometry (diaphragm edge is depicted with a dashed line).

relationship, which agrees with values reported in the literature (e.g., $35 \mu \mathrm{V} / \mathrm{K}[38])$.

We then investigated the device's transient and steady-state responses. Both chambers of the device, again heated using the microheaters, were packed with polystyrene microbeads, which were enzyme-functionalized for later metabolite measurements. The flow resistance of each chamber was measured to facilitate choice of a suitable flow rate whose associated driving pressure was sustainable by the diaphragms. The flow resistance was found to be $15.3 \mathrm{kPa} /(\mathrm{mL} / \mathrm{h})$ from the measurement. This is in general agreement with the calculated value $19.1 \mathrm{kPa} /(\mathrm{mL} / \mathrm{h})$

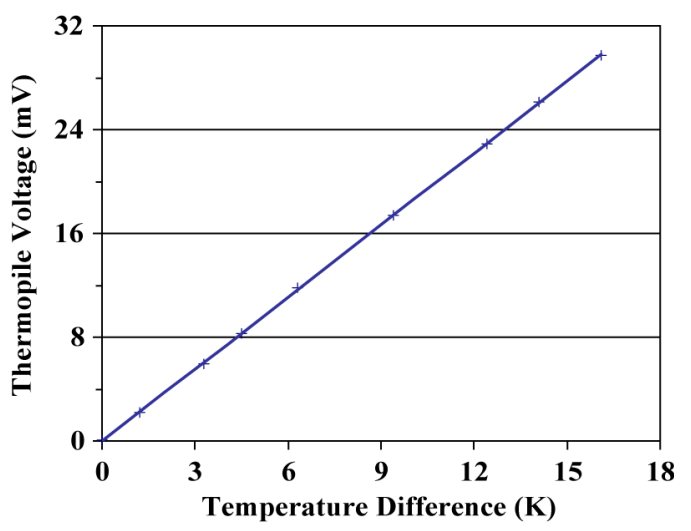

Fig. 6. Output of a fifty-junction thermopile as the temperature difference between the hot and cold junctions of the thermopile was varied. The solid line is the best linear fit to the experimental data.

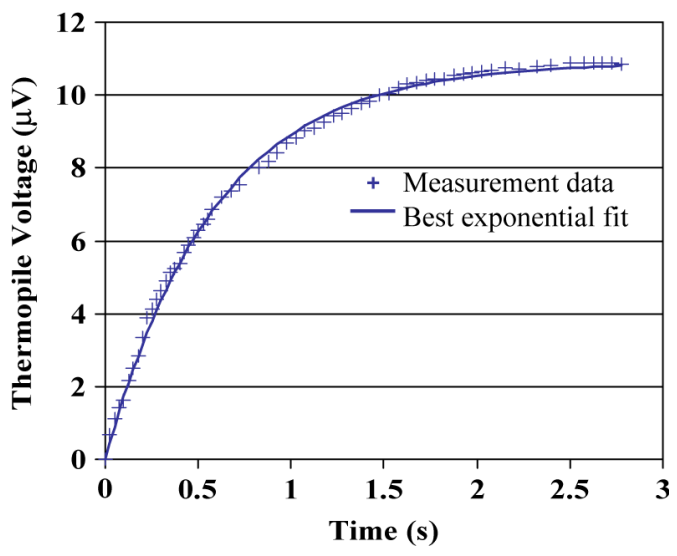

Fig. 7. Transient response when the chambers were heated with a unit-step power applied to the integrated resistive heaters.

from a packed-bed flow model [39], with the error possibly caused by the chambers not packed at full capacity with microbeads as assumed in the model. For a flow rate of $0.5 \mathrm{~mL} / \mathrm{h}$ (used later in metabolite measurements), the pressure applied to each diaphragm was thus estimated to be about $7.6 \mathrm{kPa}$.

To characterize the device's transient thermal response, the microbead-packed chambers were filled with PBS buffer, which was stationary during the measurement. A constant Joule heating power of $9.1 \mathrm{~mW}$ was suddenly applied to the sample chamber. The differential thermopile output between the two chambers was measured (Fig. 7). From these 


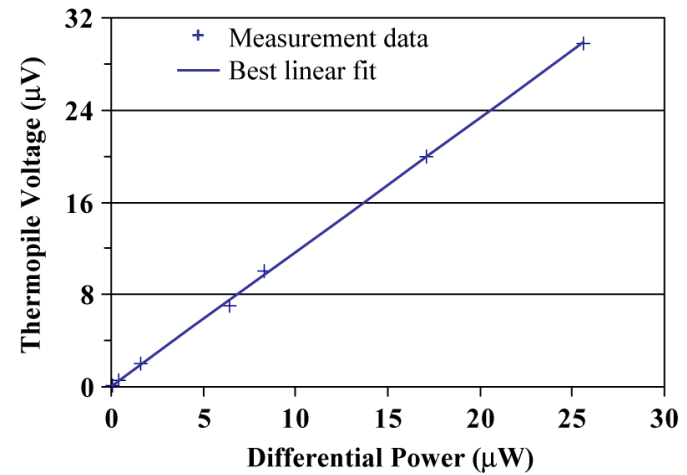

Fig. 8. Steady-state thermopile voltage corresponding to varying differential power applied via the integrated resistive heaters. The solutions were stationary in the chambers.

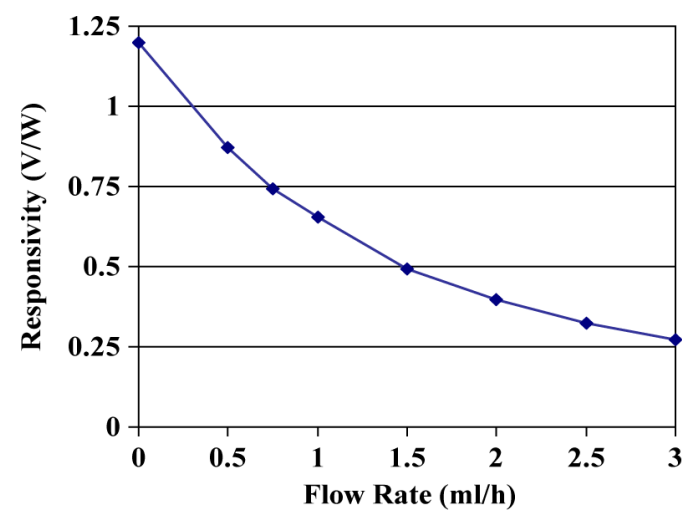

Fig. 9. Flow rate dependence of the device responsivity in flow-through mode. The experimental data points are connected to guide the eye, as is the case for all the following figures.

measurements, the thermal time constant of the device is found to be approximately $0.6 \mathrm{~s}$.

The device's responsivity in steady state heat transfer was investigated by measuring the thermopile output when the chambers were heated with different constant powers. The PBS buffer solution filling the chambers was again stationary during the measurements. The thermopile output, which is proportional to the sample-reference temperature difference, was measured as a function of the differential power between the two chambers (Fig. 8). This relationship is highly linear and its slope is the device responsivity, determined to be $K=$ $1.2 \mathrm{~V} / \mathrm{W}(8)$. The linearity suggests that nonlinear heat transfer effects (e.g., convection and radiation) were negligible within the investigated temperature range. With the device using $n=$ 50 pairs of thermocouple junctions, the thermal resistance is determined to be approximately $R_{\mathrm{th}}=649 \mathrm{~K} / \mathrm{W}$.

In relevance to flow-injection measurements, we investigated the device's steady-state responsivity when the solutions were perfused through the chambers at varying flow rates. At any given flow rate tested, the steady-state thermopile output was found to exhibit an excellent linear dependence on the differential power as applied using the microheaters. Thus, the device responsivity is independent of the differential power. On the other hand, it decreases with increasing flow rate (Fig. 9), agreeing with the intuition that the device becomes less sensitive at higher flow rates due to significant convection-induced heat

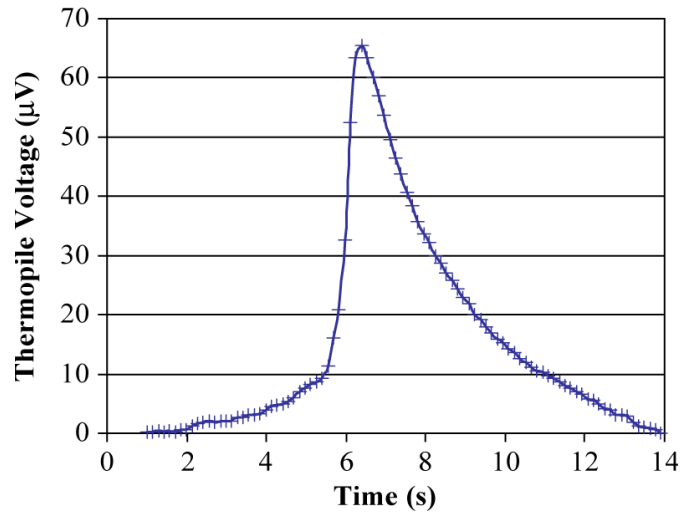

Fig. 10. Measured thermopile output as a function of time in flow-injection mode (glucose concentration: $31.3 \mathrm{mM}$ ).

losses. For the flow-through measurements below, the perfusion flow rate was chosen to be $0.5 \mathrm{~mL} / \mathrm{h}$, which corresponds to a responsivity of $0.87 \mathrm{~V} / \mathrm{W}$, or an effective thermal resistance of $470 \mathrm{~K} / \mathrm{W}$.

For both flow-injection and flow-through measurements, it is interesting to consider potential errors caused by the separation of the solution from the thermopile by the SU-8 diaphragm. With the thermal conductivity of SU-8 taken to be $0.2 \mathrm{~W} / \mathrm{m} \cdot \mathrm{K}$, it can be estimated that this separation corresponds to a thermal resistance on the order of $6 \mathrm{~K} / \mathrm{W}$, which is $0.9 \%$ and $1.6 \%$ of the effective thermal resistance between the chambers in flowinjection and flow-through modes, respectively. Therefore, it is believed that errors caused by the thermal separation of the solution from the thermopile by the SU-8 diaphragm were negligible.

\section{B. Metabolite Measurements}

The utility of the thermal biosensor for metabolic monitoring was demonstrated by measurement of glucose at physiologically relevant concentrations. The measurement is based on oxidation of glucose and decomposition of hydrogen peroxide, catalyzed respectively by the enzymes glucose oxidase and catalase immobilized on the microbeads packed in the chambers. The enzymatic reactions are as follows:

$$
\begin{aligned}
\beta-\mathrm{D}-\text { Glucose } & +\mathrm{O}_{2} \stackrel{\text { Glucose Oxidase }}{\longrightarrow} \mathrm{H}_{2} \mathrm{O}_{2} \\
& +\mathrm{p}-\mathrm{D}-\text { Gluconolactone } \\
& 2 \mathrm{H}_{2} \mathrm{O}_{2} \stackrel{\text { Catalase }}{\longrightarrow} 2 \mathrm{H}_{2} \mathrm{O}+\mathrm{O}_{2} .
\end{aligned}
$$

Note that while the measurements involved two reactions, the operating principle described in Section III is valid in general. In particular, because the reaction involving catalase is orders of magnitude faster than that involving glucose oxidase, the reaction time constant is determined by glucose oxidase. Measurement results in flow-injection and flow-through modes are presented below.

Measurements in Flow-Injection Mode: Glucose solutions with concentrations ranging from 0 to $31.3 \mathrm{mM}$ were injected into the sample chamber $(0.8 \mu \mathrm{l})$. To illustrate the thermal evolution process, Fig. 10 shows the thermopile output as a 


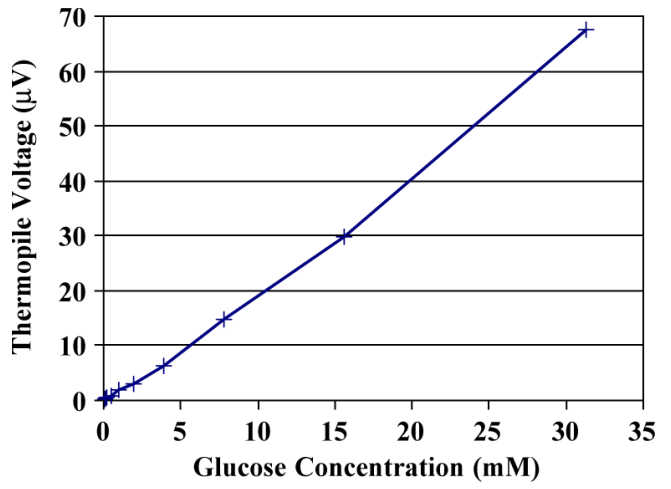

Fig. 11. Measured maximum thermopile output at varying glucose concentrations in flow-injection mode.

function of time at a glucose concentration of $31.3 \mathrm{mM}$. The voltage (or proportionally, the temperature difference between the chambers) over an initial period increases with time until it reaches the maximum value, and this period likely corresponds to the processes of chamber filling and homogenization by the injected glucose solution, as well as thermal equilibration. Following the maximum, the voltage decays in time, which reflects the decreasing glucose concentration due to the enzymatic reactions. The time scale of the reaction process, corresponding to the decay, is estimated to be about $2.5 \mathrm{~s}$, considerably larger than the device's thermal time constant $(0.6 \mathrm{~s})$ as determined above using the microheaters. Thus, the steady-state heat transfer assumption made in Section III is reasonable for these measurements. Here, while (5) (flow-injection mode) is not directly applicable since the glucose concentration is actually larger than the Michaelis constant of glucose oxidase $(\sim 20 \mathrm{mM})$, it does correctly predict the trend in the voltage decay.

We measured the maximum thermopile voltage as glucose solutions of varying concentrations were injected (Fig. 11). It can be seen that maximum voltage increases with glucose concentration almost linearly. This is again consistent with (5) and (8), which predict that the maximum voltage is proportional to the analyte concentration. The sensitivity of the thermopile voltage with respect to the glucose concentration corresponds to the slope of this linear relationship, which is determined to be approximately $2.1 \mu \mathrm{V} / \mathrm{mM}$. With the noise in the thermopile voltage measured to be about $53 \mathrm{nV}$, this sensitivity allows us to estimate the device's resolution in glucose concentration measurements to be about $0.025 \mathrm{mM}$.

1) Measurements in Flow-Through Mode: First, to illustrate the dependence of the thermopile output on flow rate, we made flow-through measurements at a fixed glucose concentration of $31.3 \mathrm{mM}$ and varying flow rates (Fig. 12). We observe that the thermopile output initially increases and then decreases with flow rates, and therefore an optimal flow rate $(0.5 \mathrm{~mL} / \mathrm{h})$ exists that maximizes the thermopile output. This can be attributed to forced convection heat transfer effects. In flow-through mode, a significant part of the reaction heat is removed by the flowing fluid via forced convection, especially at relatively large flow rates. On the other hand, a larger flow rate supplies more substrate to the reaction chamber, generating a larger thermal power. Indeed, (5) predicts that more power is supplied to the chamber at a larger flow rate, while (7) shows that effective ther-

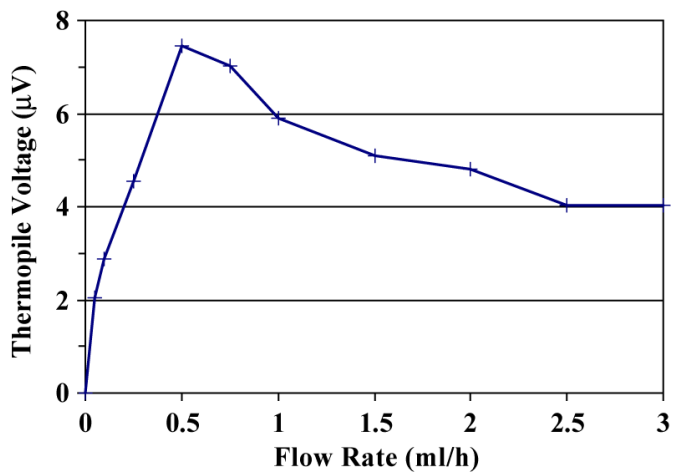

Fig. 12. Measured thermopile output in flow-through mode at varying flow rates (glucose concentration: $31.3 \mathrm{mM} / \mathrm{mL}$ ).

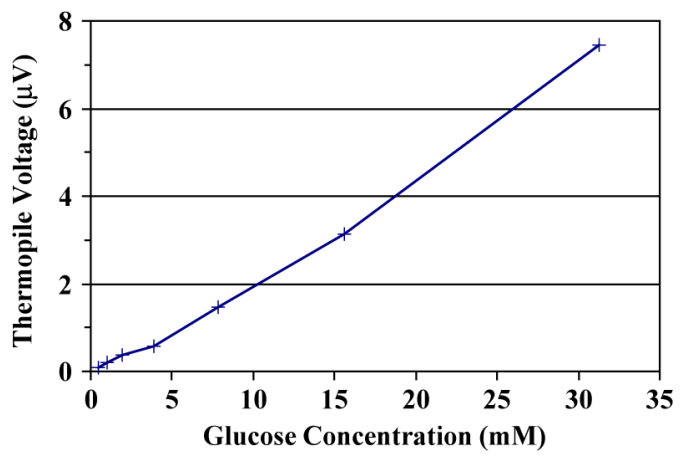

Fig. 13. Measured thermopile output in flow-through mode at varying glucose concentrations (flow rate: $0.5 \mathrm{~mL} / \mathrm{h}$ ).

mal resistance decreases with flow rate. These two competing effects result in the existence of an optimal flow rate.

Fig. 13 shows measurement results when the glucose solution was pumped through the sample chamber at a fixed flow rate of $0.5 \mathrm{~mL} / \mathrm{h}$. It can be seen that the thermopile output increases fairly linearly with the glucose concentration. This can be explained by the relatively small flow rate used in the measurements using theory described in Section III. Specifically, at the $0.5 \mathrm{~mL} / \mathrm{h}$ flow rate, the residence time of the solution in the chamber is approximately $8.6 \mathrm{~s}$, which is considerably larger than the reaction time scale (about $2.5 \mathrm{~s}$ ). Thus, (6) applies, and the reaction power is approximately linear with glucose concentration. Equation (7) then predicts that the thermopile voltage should also depend on the glucose concentration approximately linearly. The slope of this relationship provides the sensitivity of the thermopile voltage with respect to glucose concentration, which is estimated to be $0.24 \mu \mathrm{V} / \mathrm{mM}$ for the $0.5 \mathrm{~mL} / \mathrm{h}$ flow rate. Accordingly, with the thermopile noise voltage measured to be $96 \mathrm{nV}$, it is estimated that the resolution of the device in glucose concentration measurements is approximately $0.4 \mathrm{mM}$.

It is worth noting that the sensitivity in flow-through mode is almost an order of magnitude smaller than the sensitivity in the flow-injection case. This is due not only to the smaller effective thermal resistance in flow-through mode, but also the diminished flow-through total reaction power. Indeed, in (5), we see that for flow-injection mode $\lambda V$, the ratio of the chamber volume to the reaction time scale $(\sim 2.5 \mathrm{~s})$, is about $1.7 \mathrm{~mL} / \mathrm{h}$, or more than three times the perfusion flow rate 
in flow-through mode. Thus, the total reaction power in flowthrough mode is diminished by the same factor when compared with that in flow-injection mode. It should nonetheless be noted that flow-through measurements may still be of practical interest; for example, they can be more simply integrated with microdialysis-based subcutaneous glucose sampling in a metabolic monitoring microsystem.

Possible Directions of Future Work: This paper has demonstrated the promise of MEMS-based thermal biosensing for monitoring of glucose and other metabolites. From the experimental results above, we can make observations on several issues that should be addressed in future work. First, it will be interesting to investigate the specificity of the MEMS thermal biosensor. Because the enzymatic reaction is specific to glucose, thermal biosensing that is based on it should in general afford glucose-specific detection. In practice, specificity can be affected by thermal disturbances such as unspecific reactions and ambient temperature fluctuations. These disturbance effects, fortunately, should be minimized thanks to commonmode cancellation as enabled by the device's differential measurement scheme. Experiments should be conducted to evaluate the effects of such thermal disturbances on the MEMS device's capability for specific glucose detection.

Second, the MEMS thermal biosensor, based on inexpensive polymers, can potentially be made available for disposable use. Nonetheless, it will be of interest to investigate its suitability for extended use as well. This would be relevant when the device is to be included as an integral component in a metabolic monitoring microsystem that is intended to function for a prolonged period. Future work should address the chemical and mechanical stability of the device's microfluidic design, which is based on PDMS and SU-8. Our experiments have yielded evidence that PDMS and SU-8 are chemically stable for glucose sensing, but further studies are needed. Moreover, the polymeric structure's mechanical stability is a significant issue. In particular, the mechanical behavior of the SU-8 freestanding diaphragm, which must sustain pressures up to a few tens of kilopascal, will generally change with time. This is because SU-8, a polymer, is viscoelastic in nature. The viscoelasticity of SU-8 has recently been studied in the literature [40], and its impact on the MEMS device's mechanical stability should be examined. The primary goal of such studies should be to ensure the structural integrity of the device; other timedependent mechanical changes might be addressed by regular device calibration.

Third, the MEMS device's measurement resolution and repeatability need to be addressed. The estimated resolution for the present device, while generally adequate in flow-injection mode, may need improvement in flow-through mode in order for the device to be viable for practical glucose monitoring [22], [23]. This might involve using more sensitive thermopile materials [9] and more stable flow rates with minimized fluctuations [34]. Practical glucose monitoring also requires high repeatability. Our preliminary data involving multiple measurements at a given glucose concentration has yielded a repeatability better than $10 \%$. Systematic experiments should be conducted in future work to assess the device repeatability. Improvement of repeatability may be accomplished by improved design and fabrication techniques. These might involve, for example, the use of a stiffer polymeric structure (e.g., thicker freestanding polymer diaphragms) that still offers adequate thermal isolation, and more consistent measurement conditions (e.g., minimized ambient temperature variations and more regularly packed beads in the device chambers).

\section{CONCLUSION}

A MEMS thermal biosensor with integrated microfluidics has been developed for metabolic monitoring applications. The device consists of two microchambers each based on a freestanding diaphragm. Resistive microheaters are fabricated on the diaphragms, and an integrated microthermopile spans the two diaphragms. The chambers are packed with microbeads functionalized with enzymes specific to a metabolite analyte. During measurement, a sample solution containing the analyte and a reference buffer solution are respectively introduced into the chambers by either injection as a batch (flow-injection mode) or continuous perfusion (flow-through mode). The thermal power arising from enzyme-catalyzed reactions of the metabolite is detected as the differential power between the chambers. The differential measurement scheme allows highly sensitive measurement of the enzymatic reaction heat, as it affords excellent cancellation of common-mode disturbances in the two chambers.

Characterization of a fabricated MEMS thermal biosensor, with its chambers filled with buffer solutions and using integrated microheaters, has yielded responsivities of $1.2 \mathrm{~V} / \mathrm{W}$, when the buffers were stationary in the chambers, and $0.87 \mathrm{~V} / \mathrm{W}$ when the buffers were perfused through the chambers at a flow rate of $0.5 \mathrm{~mL} / \mathrm{h}$. Measurements of glucose at physiologically relevant concentrations have been performed. In flow-injection mode, the device's maximum thermopile output has shown a sensitivity of approximately $2.2 \mu \mathrm{V} / \mathrm{mM}$ with respect to glucose concentration. With a measured thermopile noise voltage of $53 \mathrm{nV}$, this yields a resolution of about $0.025 \mathrm{mM}$ for glucose measurements. In flow-through mode, the thermopile voltage is a function of flow rate, and achieves the maximum at a flow rate around $0.5 \mathrm{~mL} / \mathrm{h}$. At this flow rate, the sensitivity of the thermopile voltage to glucose concentration is estimated to be $0.24 \mu \mathrm{V} / \mathrm{mM}$ at $0.5 \mathrm{~mL} / \mathrm{h}$ perfusion rate. Based on a measured thermopile noise voltage of $96 \mathrm{nV}$, the resolution of the device in glucose concentration measurements is estimated to be $0.4 \mathrm{mM}$. These results demonstrate the promise of the MEMSbased thermal biosensor, which can potentially be integrated with subcutaneous sampling methods such as microdialysis, in a microsystem for monitoring of glucose and other metabolites in medical applications.

\section{ACKNOWLEDGMENT}

The authors would like to thank the MEMS Laboratory in the Department of Electrical and Computer Engineering, Carnegie Mellon University, for generously granting access to its fabrication and characterization facilities. The authors would also like to thank S. Zhang for his assistance in microfabrication, and Y. Yang for helpful discussions in instrumentation. 


\section{REFERENCES}

[1] B. Danielsson, "Calorimetric biosensors," Biochem. Soc. Trans., vol. 19, pp. 26-28, 1991

[2] P. Bataillard, E. Steffgen, S. Haemmerli, A. Manz, and H. M. Widmer, "An integrated silicon thermopile as biosensor for the thermal monitoring of glucose, urea and penicillin," Biosens. Bioelectron., vol. 8, no. 2, pp. 8998, 1993.

[3] R. Lenggenhager, H. Baltes, J. Peer, and M. Forster, "Thermoelectric infrared sensors by CMOS technology," IEEE Electron Device Lett., vol. 13, no. 9, pp. 454-456, Sep. 1992.

[4] A. W. Vanherwaarden, P. M. Sarro, J. W. Gardner, and P. Bataillard, "Liquid and gas micro-calorimeters for (Bio)chemical measurements," Sens. Actuators A, Phys., vol. 43, no. 1-3, pp. 24-30, May 1994.

[5] B. Xie, M. Mecklenburg, B. Danielsson, O. Ohman, and F. Winquist, "Microbiosensor based on an integrated thermopile," Anal. Chim. Acta, vol. 299, no. 2, pp. 165-170, Dec. 1994.

[6] Y. Y. Zhang and S. Tadigadapa, "Calorimetric biosensors with integrated microfluidic channels," Biosens. Bioelectron., vol. 19, no. 12, pp. 17331743, Jul. 2004.

[7] J. L. Garden, E. Chateau, and J. Chaussy, "Highly sensitive Ac nanocalorimeter for microliter-scale liquids or biological samples," Appl. Phys. Lett., vol. 84, no. 18, pp. 3597-3599, May 2004.

[8] E. A. Johannessen, J. M. R. Weaver, P. H. Cobbold, and J. M. Cooper, "Heat conduction nanocalorimeter for Pl-scale single cell measurements," Appl. Phys. Lett., vol. 80, no. 11, pp. 2029-2031, Mar. 2002.

[9] H. L. Zou, D. M. Rowe, and G. Min, "Preparation and characterization of p-type $\mathrm{Sb}_{2} \mathrm{Te}_{3}$ and n-type $\mathrm{Bi}_{2} \mathrm{Te}_{3}$ thin films grown by coevaporation," J. Vac. Sci. Technol. A, Vac. Surf. Films, vol. 19, no. 3, pp. 899-903, May 2001.

[10] S. L. Lai, G. Ramanath, L. H. Allen, and P. Infante, "Heat capacity measurements of Sn nanostructures using a thin-film differential scanning calorimeter with $0.2 \mathrm{~nJ}$ sensitivity," Appl. Phys. Lett., vol. 70, no. 1, pp. 43-45, Jan. 1997.

[11] T. A. S. Srinivas, P. J. Timans, R. J. Butcher, and H. Ahmed, "Thermal and infrared performance of a free-standing microthermocouple," Rev. Sci. Instrum., vol. 64, no. 12, pp. 3602-3611, Dec. 1993.

[12] S. L. Lai, G. Ramanath, L. H. Allen, P. Infante, and Z. Ma, "Highspeed $\left(10^{4 \circ} \mathrm{C} / \mathrm{s}\right)$ scanning microcalorimetry with monolayer sensitivity $\left(\mathrm{J} / \mathrm{m}^{2}\right), "$ Appl. Phys. Lett., vol. 67, no. 9, pp. 1229-1231, Aug. 1995.

[13] C. Hagleitner, A. Hierlemann, D. Lange, A. Kummer, N. Kerness, O. Brand, and H. Baltes, "Smart single-chip gas sensor microsystem," Nature, vol. 414, no. 6861, pp. 293-296, Nov. 2001.

[14] C. Hagleitner, D. Lange, A. Hierlemann, O. Brand, and H. Baltes, "CMOS single-chip gas detection system comprising capacitive, calorimetric and mass-sensitive microsensors," IEEE J. Solid-State Circuits, vol. 37, no. 12, pp. 1867-1878, Dec. 2002.

[15] B. Xie, K. Ramanathan, and B. Danielsson, "Mini/micro thermal biosensors and other related devices for biochemical/clinical analysis and monitoring," TrAC-Trends Anal. Chem., vol. 19, no. 5, pp. 340-349, May 2000.

[16] M. Shimohigoshi and I. Karube, "Development of uric acid and oxalic acid sensors using a bio-thermochip," Sens. Actuators B, Chem., vol. 30, no. 1, pp. 17-21, Jan. 1996.

[17] M. Mecklenburg, C. Lindbladh, H. S. Li, K. Mosbach, and B. Danielsson, "Enzymatic amplification of a flow-injected thermometric enzyme-linked immunoassay for human insulin," Anal. Biochem., vol. 212, no. 2, pp. 388-393, Aug. 1993.

[18] K. Verhaegen, K. Baert, J. Simaels, and W. Van Driessche, "A highthroughput silicon microphysiometer," Sens. Actuators A, Phys., vol. 82, no. 1-3, pp. 186-190, May 2000.

[19] I. Wadso, "Isothermal microcalorimetry. Current problems and prospects," J. Therm. Anal. Calorim., vol. 64, no. 1, pp. 75-84, Apr. 2001.

[20] A. Wolf, A. Weber, R. Huttl, J. Lerchner, and G. Wolf, "Sequential flow injection analysis of complex systems using calorimetric detection," Thermochim. Acta, vol. 382, no. 1/2, pp. 89-98, Jan. 2002.

[21] B. Xie, M. Mecklenburg, B. Danielsson, O. Ohman, P. Norlin, and F. Winquist, "Development of an integrated thermal biosensor for the simultaneous determination of multiple analytes," Analyst, vol. 120, no. 1, pp. 155-160, 1995.

[22] DexCom, Inc., Dexcom STS System. [Online]. Available: http://www. dexcom.com/

[23] Medtronic MiniMed, Guardian Real-Time Continuous Glucose Monitoring System. [Online]. Available: http://www.minimed.com/products/ guardian/index.html
[24] B. Xie, B. Danielsson, P. Norberg, F. Winquist, and I. Lundstrom, "Development of a thermal micro-biosensor fabricated on a silicon chip," Sens. Actuators B, Chem., vol. 6, no. 1-3, pp. 127-130, Jan. 1992.

[25] R. Kurita, K. Hayashi, X. Fan, K. Yamamoto, T. Kato, and O. Niwa, "Microfluidic device integrated with pre-reactor and dual enzyme-modified microelectrodes for monitoring in vivo glucose and lactate," Sens. Actuators B, Chem., vol. 87, no. 2, pp. 296-303, Dec. 2002.

[26] J. Wu, J. Suls, and W. Sansen, "The glucose sensor integratable in the microchannel," Sens. Actuators B, Chem., vol. 78, no. 1-3, pp. 221-227, Aug. 2001.

[27] J. Wang, M. P. Chatrathi, B. M. Tian, and R. Polsky, "Microfabricated electrophoresis chips for simultaneous bioassays of glucose, uric acid, ascorbic acid, and acetaminophen," Anal. Chem., vol. 72, no. 11, pp. 2514-2518, Jun. 2000.

[28] C. Pu, Z. H. Zhu, and Y. H. Lo, "A surface-micromachined optical selfhomodyne polarimetric sensor for noninvasive glucose monitoring," IEEE Photon. Technol. Lett., vol. 12, no. 2, pp. 190-192, Feb. 2000.

[29] M. Paranjape, J. Garra, S. Brida, T. Schneider, R. White, and J. Currie, "A PDMS dermal patch for non-intrusive transdermal glucose sensing," Sens. Actuators A, Phys., vol. 104, no. 3, pp. 195-204, May 2003.

[30] M. Lei, A. Baldi, E. Nuxoll, R. A. Siegel, and B. Ziaie, "A hydrogelbased implantable micromachined transponder for wireless glucose measurement," Diabetes Technol. Ther, vol. 8, no. 1, pp. 112-122, Feb. 2006.

[31] Y. Zhao, S. Li, A. Davidson, B. Yang, Q. Wang, and Q. Lin, "A MEMS viscometric sensor for continuous glucose monitoring," J. Micromech. Microeng., vol. 17, no. 12, pp. 2528-2537, Dec. 2007.

[32] J. D. Zahn, D. Trebotich, and D. Liepmann, "Microdialysis microneedles for continuous medical monitoring," Biomed. Microdevices, vol. 7, no. 1, pp. 59-69, Mar. 2005.

[33] Y. C. Hsieh and J. D. Zahn, "Glucose recovery in a microfluidic microdialysis biochip," Sens. Actuators B, Chem., vol. 107, no. 2, pp. 649-656, Jun. 2005.

[34] B. Yang, G. C. Lopez, A. J. Rosenbloom, and Q. Lin, "Design and rapid fabrication of a microfluidic fraction collector," Int. J. Nonlinear Sci. Numer. Simul., vol. 3, no. 3/4, pp. 267-272, Aug. 2002.

[35] J. M. Berg, J. L. Tymoczko, and L. Stryer, Biochemistry, 5th ed. New York: Freeman, 2002.

[36] A. Wolf, A. Weber, R. Huttl, J. Lerchner, and G. Wolf, "Sequential flow injection analysis based on calorimetric detection," Thermochim. Acta, vol. 337, no. 1, pp. 27-38, Oct. 1999.

[37] Y. N. Xia and G. M. Whitesides, "Soft lithography," Angew. Chem.-Int. Edition, vol. 37, pp. 551-575, 1998.

[38] D. M. Rowe, CRC Handbook of Thermoelectrics. Boca Raton, FL: CRC Press, 1995.

[39] D. A. Nield and A. Bejan, Convection in Porous Media, 2nd ed. New York: Springer-Verlag, 1999.

[40] K. I. Schiffmann and C. Brill, "Testing the viscoelastic properties of SU8 photo resist thin films at different stages of processing by nanoindentation creep and stress relaxation," Int. J. Mater. Res., vol. 98, no. 5, pp. 397403, 2007.

Li Wang received the B.S. and M.S. degrees in mechanical engineering from Tsinghua University, Beijing, China, in 1999 and 2002, respectively, and the Ph.D. degree in mechanical engineering from Carnegie Mellon University, Pittsburgh, PA, in 2007.

$\mathrm{He}$ is currently with the Department of Mechanical Engineering, Carnegie Mellon University. His research interests include miniaturized devices with integrated microfluidics for biomedical applications.

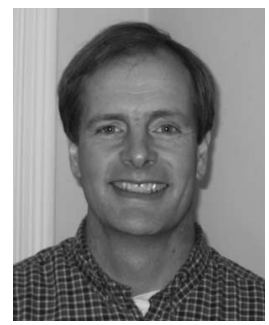

David M. Sipe received the B.S. degree in chemistry from Stanford University, Stanford, CA, in 1981, and the Ph.D. degree in biological sciences/biochemistry from Carnegie Mellon University, Pittsburgh, PA, in 1990.

He was with Becton-Dickinson in California. He was a Postdoctoral Researcher in molecular biology at the University of Utah, Salt Lake City, from 1990 to 1992 , later holding various positions in academia and industry in Utah. He is currently a Postdoctoral Researcher in chemistry at Carnegie Mellon. His interests include ultra high mass spectrometry to characterize proteins, nucleic acids, biomolecular complexes, and nanoparticles, as well as immunoassays. 


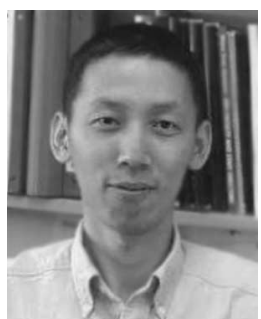

Yong Xu (S'99-M'02) received the B.S. degree in electronics engineering from Tsinghua University, Beijing, China, in 1997, and the M.S. and Ph.D. degrees in electrical engineering from California Institute of Technology, Pasadena, in 1998 and 2002, respectively.

He is currently an Assistant Professor in the Department of Electrical and Computer Engineering, Wayne State University, Detroit, MI. His research interests include MEMS smart skins, intelligent textiles, microfluidics, biosensors, medical devices, novel packaging technology, and nanotechnology.

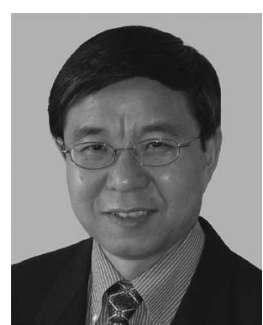

Qiao Lin received the Ph.D. degree in mechanical engineering from California Institute of Technology (Caltech), Pasadena, in 1998, with thesis research in robotics.

He conducted postdoctoral research in microelectromechanical systems (MEMS) at the Caltech Micromachining Laboratory from 1998 to 2000, and was an Assistant Professor of mechanical engineering at Carnegie Mellon University, Pittsburgh, PA, from 2000 to 2005. He has been an Associate Professor of mechanical engineering at Columbia University, New York, NY, since 2005. His research interests are in designing and creating integrated micro/nanosystems, in particular, MEMS and microfluidic systems, for biomedical applications. 